\title{
AN INEQUALITY FOR THE DISTRIBUTION OF A SUM OF CERTAIN BANACH SPACE VALUED RANDOM VARIABLES
}

\author{
BY J. KUELBS ${ }^{1}$
}

Communicated by Jacob Feldman, September 29, 1973

1. Introduction. Throughout the paper $B$ is a real separable Banach space with norm $\|\cdot\|$, and all measures on $B$ are assumed to be defined on the Borel subsets of $B$. We denote the topological dual of $B$ by $B^{*}$.

A measure $\mu$ on $B$ is called a mean zero Gaussian measure if every continuous linear function $f$ on $B$ has a mean zero Gaussian distribution with variance $\int_{B}[f(x)]^{2} \mu(d x)$. The bilinear function $T$ defined on $B^{*}$ by

$$
T(f, g)=\int_{B} f(x) g(x) \mu(d x) \quad\left(f, g \in B^{*}\right)
$$

is called the covariance function of $\mu$. It is well known that a mean zero Gaussian measure on $B$ is uniquely determined by its covariance function.

However, a mean zero Gaussian measure $\mu$ on $B$ is also determined by a unique subspace $H_{\mu}$ of $B$ which has a Hilbert space structure. The norm on $H_{\mu}$ will be denoted by $\|\cdot\|_{\mu}$ and it is known that the $B$ norm $\|\cdot\|$ is weaker than $\|\cdot\|_{\mu}$ on $H_{\mu}$. In fact, $\|\cdot\|$ is a measurable norm on $H_{\mu}$ in the sense of [3]. Since $\|\cdot\|$ is weaker than $\|\cdot\|_{\mu}$ it follows that $B^{*}$ can be linearly embedded into the dual of $H_{\mu}$, call it $H_{\mu}^{*}$, and identifying $H_{\mu}$ with $H_{\mu}^{*}$ in the usual way we have $B^{*} \subseteq H_{\mu} \subseteq B$. Then by the basic result in [3] the measure $\mu$ is the extension of the canonical normal distribution on $H_{\mu}$ to $B$. We describe this relationship by saying $\mu$ is generated by $H_{\mu}$. For details on these matters as well as additional references see [3] and [4].

2. The basic inequality. The norm $\|\cdot\|$ on $B$ is twice directionally differentiable on $B-\{0\}$ if for $x, y \in B, x+t y \neq 0$, we have

$$
(d / d t)\|x+t y\|=D(x+t y)(y)
$$

AMS (MOS) subject classifications (1970). Primary 60B05, 60B10, 60F10; Secondary 28A40.

Key words and phrases. Measurable norm, Gaussian measure, law of the iterated logarithm, central limit theorem, differentiable norm.

${ }^{1}$ Supported in part by NSF Grant GP 18759.

Copyright (c) American Mathematical Society 1974 
where $D: B-\{0\} \rightarrow B^{*}$ is measurable from the Borel subsets of $B$ generated by the norm topology to the Borel subsets of $B^{*}$ generated by the weakstar topology, and

$$
\left(d^{2} / d t^{2}\right)\|x+t y\|=D_{x+t y}^{2}(y, y)
$$

where $D_{x}^{2}$ is a bounded bilinear form on $B \times B$. We call $D_{x}^{2}$ the second directional derivative of the norm, and without loss of generality we can assume $D_{x}^{2}$ is a symmetric bilinear form. That is, if $T_{x}$ is a bilinear form which satisfies $(2.2)$ then $\Lambda_{x}(y, z)=\left[T_{x}(y, z)+T_{x}(z, y)\right] / 2$ also satisfies (2.2) and $\Lambda_{x}$ is symmetric. Hence in all that follows we assume $D_{x}^{2}$ is a symmetric bilinear form. Of course, if the norm is actually twice Fréchet differentiable on $B$ with second derivative at $x$ given by $\Lambda_{x}$, then it is well known that $\Lambda_{x}$ is a symmetric bilinear form on $B \times B$, and in this case $D_{x}^{2}$ would be equal to $\Lambda_{x}$ since symmetric bilinear forms are uniquely determined on the diagonal of $B \times B$.

If $D_{x}^{2}(y, y)$ is continuous in $x(x \neq 0)$ and for all $r>0$ and $x, h \in B$ such that $\|x\| \geqq r$ and $\|h\| \leqq r / 2$ we have

$$
\left|D_{x+h}^{2}(h, h)-D_{x}^{2}(h, h)\right| \leqq C_{r}\|h\|^{2+\alpha}
$$

for some fixed $\alpha>0$ and some constant $C_{r}$ we say the second directional derivative is $\operatorname{Lip}(\alpha)$ away from zero.

We now can state our main result.

THEOREM 2.1. Let B denote a real separable Banach space with norm $\|\cdot\|$. Let $\|\cdot\|$ be twice directionally differentiable on $B$ with the second derivative $D_{x}^{2}$ being $\operatorname{Lip}(\alpha)$ away from zero for some $\alpha>0$ and such that $\sup _{\|x\|=1}\left\|D_{x}^{2}\right\|<\infty$. Let $X_{1}, X_{2}, \cdots$ be independent $B$-valued random variables such that for some $\delta>0$

$$
\sup _{k} E\left\|X_{k}\right\|^{2+\delta}<\infty, \quad E X_{k}=0 \quad(k=1,2, \cdots)
$$

and having common covariance function $T(f, g)=E\left(f\left(X_{k}\right) g\left(X_{k}\right)\right)\left(f, g \in B^{*}\right)$. Then, if $T$ is the covariance function of a mean zero Gaussian measure $\mu$ on $B$, it follows for $t \geqq 0$ and any $\beta>0$ that

$$
P\left(\left\|\frac{X_{1}+\cdots+X_{n}}{\sqrt{ } n}\right\| \geqq t\right) \leqq 2 \mu(x:\|x\| \geqq t-\beta)+O\left(n^{-\min (\alpha, \delta) / 2}\right)
$$

where the bounding constant is uniform in $t \geqq 2 \beta$.

The proof of Theorem 2.1 uses a method which is due to Trotter [7]. The application of Trotter's method in this setting depends on a number of important relationships between $H_{\mu}$ and $B$ as well as some of the 
nontrivial properties of Gaussian measures on $B$. The details of the proof are lengthy and will be presented in [6].

3. Applications of the basic inequality. Using the inequality of Theorem 2.1 we can obtain the central limit theorem and the law of the iterated logarithm for a sequence of $B$-valued random variables.

THEOREM 3.1. Let $B$ and $\left\{X_{k}\right\}$ satisfy the conditions in Theorem 2.1, and assume $\mu$ is a Gaussian measure on $B$ with covariance function $T$. Then, if $\mu_{n}$ denotes the measure induced on $B$ by $\left(X_{1}+\cdots+X_{n}\right) / \sqrt{ } n$, we have $\lim _{n} \mu_{n}=\mu$ in the sense of weak convergence.

The proof of Theorem 3.1 is not difficult and the main idea is to use (2.5) to prove that for each $\varepsilon>0$ there is a finite dimensional subspace $E$ of $B$ such that

$$
\mu_{n}\left(E^{\varepsilon}\right)>1-\varepsilon \quad(n \geqq 1) .
$$

Here $E^{\varepsilon}$ is the $\varepsilon$ neighborhood of $E$ in $B$. Since the finite dimensional distributions of the sequence $\left\{\mu_{n}\right\}$ converge to those of $\mu,(3.1)$ is then sufficient for the conclusion of Theorem 3.1.

We now turn to the law of the iterated logarithm. $L L n$ denotes $\log \log n$ if $n \geqq 3$ and 1 for $n=1,2$.

THEOREM 3.2. Let $B$ and $\left\{X_{k}\right\}$ satisfy the conditions in Theorem 2.1, and assume $\mu$ is a Gaussian measure on B with covariance function $T$. If $K$ is the unit ball of the Hilbert space $H_{\mu}$ which generates $\mu$, then

$$
P\left(\lim _{n}\left\|\frac{X_{1}+\cdots+X_{n}}{(2 n L L n)^{1 / 2}}-K\right\|=0\right)=1
$$

and

$$
P\left(C\left(\left\{\frac{X_{1}+\cdots+X_{n}}{(2 n L L n)^{1 / 2}}\right\}\right)=K\right)=1
$$

where $C\left(\left\{a_{n}\right\}\right)$ denotes the cluster set of the sequence $\left\{a_{n}\right\}$.

It is known that $K$ is a compact subset of $B$; thus (3.2) implies that with probability one the sequence $\left\{\left(X_{1}+\cdots+X_{n}\right) /(2 n L L n)^{1 / 2}\right\}$ is conditionally compact in $B$.

The proofs of (3.2) and (3.3) rest heavily on the inequality (2.5) and also on some of the nontrivial properties of Gaussian measures on $B$. The details will be given in [6].

Strassen's functional form of the law of the iterated logarithm for $B$-valued random variables can also be proved in this setting using (2.5) and the techniques developed in [5] where $B$ was assumed to be a real separable Hilbert space. 
4. Some spaces with smooth norm. Here we provide some examples of Banach spaces to which the above results apply. $(S, \Sigma, m)$ denotes a measure space and $m$ is a positive measure on $(S, \Sigma)$.

THEOREM 4.1. If $p \geqq 2$ and if for $x \in L^{p}(S, \Sigma, m)$ we define $\|x\|=$ $\left\{\int_{S}|x(s)|^{p} m(d s)\right\}^{1 / p}$, then the norm $\|\cdot\|$ has two directional derivatives and the second derivative is $\operatorname{Lip}(\alpha)$ away from zero with $\alpha=1$ for $p=2$ or $p \geqq 3$ and $\alpha=p-2$ for $2<p<3$. Furthermore, $\sup _{\|x\|=1}\left\|D_{x}^{2}\right\| \leqq 2(p-1)$.

The results of Theorem 4.1 are suggested by those in [1], but do not seem to be immediate corollaries of [1]. Their proof, however, is rather straightforward. Furthermore, the derivatives in Theorem 4.1 are actually Fréchet derivatives.

Using Theorem 4.1 and assuming $(S, \Sigma, m)$ is a $\sigma$-finite measure space we see that the $L^{p}$ spaces $(2 \leqq p<\infty)$ satisfy the conditions used above. Thus the central limit theorem and the law of the iterated logarithm are valid in these spaces. A central limit theorem for random variables with values in an $L^{p}$ space $(2 \leqq p<\infty)$ was previously known and appears in [2], but the $\log \log$ law for non-Gaussian random variables is new for $p>2$.

\section{BIBLIOGRAPHY}

1. R. Bonic and J. Frampton, Smooth functions on Banach manifolds, J. Math. Mech. 15 (1966), 877-898. MR 33 \#6647.

2. R. Fortet and $\mathrm{E}$. Mourier, Les fonctions aléatoires comme éléments aléatoires dans les espace de Banach, Studia Math. 15 (1955), 62-79. MR 19, 1202.

3. L. Gross, Abstract Wiener measure and infinite dimensional potential theory, Lectures in Modern Analysis and Applications, II, Lecture Notes in Math., vol. 140, Springer, Berlin, 1970, pp. 84-116. MR 42 \#457.

4. J. Kuelbs, Some results for probability measures on linear topological vector spaces with an application to Strassen's log log law, J. Functional Analysis 14 (1973), 28-43.

5. - Berry-Essen estimates in Hilbert space and an application to the law of the iterated logarithm, Ann. Probability (to appear).

6. - An inequality for the distribution of a sum of certain Banach space valued random variables, Studio Math. (to appear).

7. H. F. Trotter, An elementary proof of the central limit theorem, Arch. Math. 10 (1959), 226-234. MR 21 \#7559.

Department of Mathematics, University of Wisconsin, Madison, Wisconsin 53706 